\title{
Une médecine fondée sur les données probantes à l'époque de la COVID-19
}

\author{
par Cynthia A. Jackevicius
}

La médecine basée sur les données probantes se situe à la croisée des meilleures preuves cliniques disponibles, de l'expertise des cliniciens et des valeurs des patients ${ }^{1}$. Pendant la pandémie de COVID-19, nous avons manqué de données probantes de qualité à cause du manque d'essais cliniques et de la rareté de l'expertise des cliniciens, étant donné l'expérience limitée relative à l'entité inconnue de la COVID19. L'absence d'une base d'éléments probants associée à des données en constante mutation est à l'origine d'une grande incertitude, qui a entraîné parfois des prises de décision fondées sur l'émotion plutôt que sur des éléments probants.

Au début de la pandémie de COVID-19, les décisions cliniques s'appuyaient sur des preuves indirectes (p. ex., sur des études animales ou sur l'expérience du H1N1 d'il y a dix ans) et sur quelques études ou séries de cas disponibles en provenance de Chine, où la COVID-19 est apparue en premier. La base d'éléments probants s'est ensuite élargie pour y inclure des études observationnelles provenant des "points chauds " subséquents : Seattle, Washington et l'Italie. Plus tard, il y a eu une surabondance de preuves à mesure de l'apparition d'une énorme quantité d'études observationnelles, dont beaucoup figuraient dans des « moteurs de prépublication » (p. ex., www.medRxiv.org) sans avoir fait l'objet d'un examen par des pairs. Enfin, des essais contrôlés aléatoires ont été menés partout dans le monde à une vitesse record. Bien que l'établissement de ces preuves puisse être une expérience désagréable pour les cliniciens comme pour le grand public, c'est précisément de cette manière que fonctionne la médecine fondée sur les données probantes : avec une base d'éléments probants en constante évolution, suivie par des preuves de meilleure qualité et une plus grande certitude avec le temps.

Cette accélération de la production de preuves a remis en question notre capacité à garder la cadence et a amplifié les failles de nos méthodes actuelles relatives à la synthèse des preuves. Si la COVID-19 nous a enseigné quelque chose, c'est bien l'importance des données probantes et des compétences en matière de médecine factuelle dans notre processus de prise de décision clinique. Avec les nouvelles données probantes qui émergent chaque jour, la COVID-19 a permis la mise en valeur de l'apprentissage continu et l'importance de ne pas laisser stagner nos connaissances. Jamais auparavant la nécessité de se tenir au courant des nouveaux éléments de preuve et de les évaluer de manière objective n'a été aussi évidente afin que les décisions prises en collaboration avec les patients soient optimales, individualisées et appuyées par des preuves. La grande incertitude relative aux éléments de preuve a également permis de mettre en exergue l'importance d'intégrer les valeurs et les préférences des patients pour équilibrer les risques et les avantages potentiels des thérapies, même dans un environnement marqué par de plus grandes tensions entre les valeurs sociétales et individuelles.

Le raz-de-marée des données probantes liées à la COVID-19 a intensifié la course à l'innovation et la créativité dans le champ de la médecine fondée sur les preuves. La COVID-19 a imposé des changements dans des domaines où on aurait toujours souhaité un développement plus poussé. Alors que la pandémie a généré l'intérêt du public pour la science, l'évolution rapide des éléments de preuve a généré des messages incohérents qui ont semé la confusion chez les gens. Malgré les appels lancés depuis longtemps pour faire avancer la manière dont les preuves sont présentées aux " consommateurs de preuves », il est clair désormais que nous avons besoin d'un système coordonné pour organiser les données de base massives qui connaissent un rapide développement. Un raffinement des méthodes d'examen systématiques et rapides a eu lieu, y compris la prise d'initiatives pour mettre l'information à la portée du public, et les examens systématiques « vivants » et une carthographie des recommandations (https://covid19.evidenceprime.ca/) actualisés à mesure del'apparition de nouvelles preuves se sont multipliés ${ }^{2-5}$. La collaboration entre les cliniciens spécialistes des essais, qui menaient des méta-analyses prospectives d'essais cliniques en cours, en synthétisant les données probantes au fur et à mesure de leur création sont d'autres innovations apportées à la synthèse des preuves ${ }^{6}$. Enfin, le développement d'initiatives liées à l'intelligence artificielle ont permis de déterminer les preuves en évolution et de les synthétiser ${ }^{7}$. Alors que ces nouvelles initiatives sont motivées 
par l'urgence visant à comprendre les éléments probants liés à la COVID-19, il faut espérer qu'elles donneront aussi une impulsion à l'établissement de normes plus élevées quant à la manière de synthétiser l'ensemble des données probantes relatives à la COVID-19 et à toute autre maladie, pour qu'elles soient disponibles aux cliniciens et qu'elles servent également à renforcer le leadership en matière de gestion des données probantes. En ces temps marqués par l'incertitude liée à la pandémie de COVID-19, les principes de base de la médecine fondée sur les données probantes et la revitalisation de leur évolution sont plus importants que jamais.

[Traduction par l'éditeur]

\section{Références}

1. Djulbegovic B, Guyatt GH. Progress in evidence-based medicine: a quarter century on. Lancet. 2017;390(10092):415-23.

2. Oxford COVID-19 evidence service. Centre for Evidence-Based Medicine; [page consulté le 20 mars 2020]. [en ligne] : https://www.cebm. net/oxford-covid-19-evidence-service/

3. Meta-evidence-COVID-19: live meta-analysis and evidence synthesis of therapies for COVID19. Centre Hospitalier Universitaire de Lyon, Hospices Civils de Lyon, Service de Pharmacologie et de Toxicologie; [page consulté le 2020 juin 7]. [en ligne] : http://www.metaevidence.org/ COVID19.aspx
4. COVID-NMA: COVID-19 open living evidence synthesis to inform decision. Cochrane Collaboration et Organisation mondiale de la Santé ; [page consulté $1^{\text {er }}$ novembre 2020]. [en ligne] : https://covid-nma.com/

5. Siemieniuk RAC, Bartoszko JJ, Ge L, Zeraatkar D, Izcovich A, Kum E, et al. Drug treatments for covid-19: living systematic review and meta-analysis. BMJ. 2020;370:m2980.

6. WHO Rapid Evidence Appraisal for COVID-19 Therapies (REACT) Working Group. Association between administration of systemic corticosteroids and mortality among critically ill patients with COVID-19: a meta-analysis. JAMA. 2020;324(13):1330-41.

7. Brainard J. Scientists are drowning in COVID-19 papers. Can new tools keep them afloat? Science. 2020;doi:10.1126/science.abc7839.

Cynthia A. Jackevicius, BScPhm, PharmD, MSc, est professeure au département de pratique de la pharmacie, Western University of Health Sciences, Pomona, California, et scientifique auxiliaire principale à ICES, Toronto, Ontario. Elle est également rédactrice adjointe au Journal canadien de la pharmacie hospitalière. Conflits d'intérêts : Aucune déclaration.

Adresse de correspondance :

Dre Cynthia A. Jackevicius

College of Pharmacy

Western University of Health Sciences

$309 \mathrm{E}$ Second Street

Pomona CA 909469-5527 USA

Courriel : cjackevicius@westernu.edu

\section{BEST.mis better}

\section{One resource for all types of compounding by pharmacies}

\section{WHAT'S INSIDE?}

- Information for pharmacists, pharmacy technicians, planners, architects, engineers-and others who are involved in decisions or activities that affect compounding

- Guidelines for aseptic compounding, non-aseptic compounding, and compounding which involves hazardous drugs-including radiopharmaceuticals

- Best and leading guidelines on topics such as training, planning and designing the physical environment, developing an air quality strategy, cleaning and decontaminating areas, monitoring the environment, garbing and hand hygiene, developing compounding procedures, documenting, and much more-all in only 230 pages
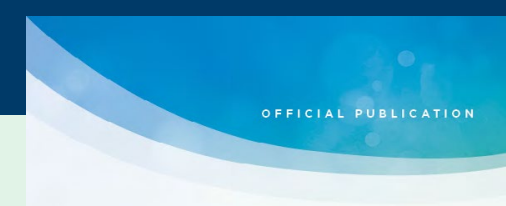

Compounding: Guidelines for Pharmacies

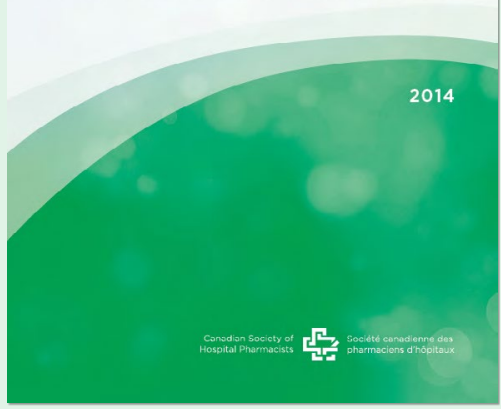

Learn what best looks like: add this publication to your library!
HAVE A SNEAK PEEK OR ORDER AT:

https://www.cshp.ca/compounding-guidelines-pharmacies CSHP MEMBERS PAY A DISCOUNTED PRICE
Canadian Society of Hospital Pharmacists

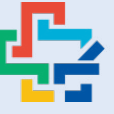

Société canadienne des pharmaciens d'hôpitaux 